\title{
Forschungsbeiträge
}

\author{
Matthias Pollmann-Schult
}

\section{Wenn Männer Väter werden - Über die Auswirkungen der Vaterschaft auf Freizeit, Lebenszufriedenheit und familiäre Beziehungen ${ }^{1}$}

\author{
When men become fathers - The effects of fatherhood on leisure \\ activities, life satisfaction, and family relations
}

\begin{abstract}
Zusammenfassung:
Der vorliegende Beitrag analysiert die Auswirkungen der Vaterschaft auf verschiedene Aspekte des Alltagshandelns. Die empirischen Analysen auf Basis des Sozio-oekonomischen Panels (SOEP) zeigen positive Effekte der Vaterschaft hinsichtlich der Lebenszufriedenheit, des bürgerschaftlichen Engagements, der religiösen Partizipation sowie der Beziehung zur Herkunftsfamilie. Diese Befunde ergänzen und erweitern frühere Erkenntnisse, die vor allem auf nachteilige Auswirkungen der Elternschaft - wie die Zunahme psychischer Belastungen, den Rückgang der Beziehungsqualität und Partnerschaftszufriedenheit sowie Einschränkungen im Freizeitverhalten - aufmerksam machen. Eine Differenzierung zwischen biologischen und sozialen Vätern zeigt, dass sich beide Vätertypen in ihrer sozialen und religiösen Partizipation sowie der Beziehung zu den eigenen Eltern voneinander unterscheiden.
\end{abstract}

Schlagwörter: Vaterschaft, Stiefvaterschaft, Lebenszufriedenheit, Freizeitaktivitäten, familiale Beziehungen.

\begin{abstract}
:
This study uses data from the German SocioEconomic Panel (SOEP) to analyze the effect of fatherhood on different aspects of the everyday life of men. The results indicate that fatherhood positively affects men's life satisfaction, civic engagement, religious participation, and the closeness of the relationship between men and their families of origin. These findings extend past research, which primarily called attention to the negative effects of fatherhood, such as increased psychological strain, reduced marital satisfaction and limitations in leisure activities. Distinguishing between biological fathers and stepfathers shows that the effect of fatherhood differs between both types of fathers with respect to their civic and religious engagements as well as to their relation to their parents.
\end{abstract}

Key words: Fatherhood, stepfatherhood, life satisfaction, leisure activities, family relations.

1 Ich bedanke mich bei Edith Busse und zwei anonymen Gutachtern für ihre hilfreichen Kommentare. 


\section{Einleitung}

Der Übergang zur Vaterschaft bringt einschneidende Veränderungen des Alltagshandelns und der Lebensführung mit sich. Wer die einschlägige Literatur zur Elternschaft sichtet, gewinnt schnell den Eindruck, dass der Übergang zur Vaterschaft vor allem ein Belastungserlebnis für die Partnerschaft darzustellen scheint, das „,bewältigt“ werden muss. Zwar wird der Übergang zur Elternschaft allgemein als „freudiges Ereignis“ (Reichle 2002) charakterisiert, jedoch fokussieren insbesondere familienpsychologische Studien auf die hieraus resultierenden Belastungen, die den Eltern etliche Übergangs- und Anpassungsleistungen abverlangen und die Partnerschaftszufriedenheit nachhaltig schmälern (Werneck 1997; ElGiamal 1997; Schneider/Rost 1999; Reichle/Werneck 1999; Reichle 2002; Kalicki et al. 2006). Auch weist die Familienforschung verstärkt auf die Beeinträchtigung der Freizeitgestaltung und deren Konsequenzen hin: Junge Eltern verbringen einen Großteil ihrer Freizeit zu Hause, wodurch sich außerhäusliche Sozialkontakte verringern und die Teilnahme am kulturellen Leben nachlässt (Rost/Schneider 1995; Vaskovics et al. 2000; Werneck 1997).

Dagegen bleiben positive Auswirkungen von Vaterschaft in den vorhandenen Studien häufig unbeachtet, obwohl die Familiengründung ganz offensichtlich nicht ausschließlich ein psychisch und physisch belastendes Ereignis darstellt, sondern ebenfalls das Leben der Eltern auf vielfältige Art bereichert. So ist zum einen ein - in der soziologischen Familienforschung bislang kaum thematisierter - positiver Effekt der Vaterschaft auf das subjektive Wohlbefinden zu erwarten. Da die Motivation zur Familiengründung insbesondere im psychischen Nutzen des Kindes für die Eltern liegt, ist anzunehmen, dass die Lebenszufriedenheit im Zuge der Familiengründung ansteigt. Auch bezüglich des Freizeitverhaltens sollten sich nicht allein restringierende Folgen der Vaterschaft zeigen. Durch die Elternschaft erfolgt zwar einerseits eine stärkere Fokussierung auf das Familienleben, andererseits bietet und erfordert die Elternschaft oftmals eine stärkere gesellschaftliche Partizipation.

Der vorliegende Beitrag analysiert die Auswirkungen der Vaterschaft auf die Lebenszufriedenheit, auf verschiedene Freizeitaktivitäten sowie auf die Beziehung zur Herkunftsfamilie und erweitert den Forschungsstand in mehrfacher Hinsicht.

Frühere Untersuchungen fokussieren aus datentechnischen Gründen zumeist auf die ersten drei bis fünf Jahre nach der Familiengründung, wodurch die Situation der Eltern mit Kindern im Schulalter unbeachtet bleibt. So ist die in der frühen Familienphase beobachtete „Verhäuslichung“ der Freizeit angesichts des hohen Betreuungsaufwands bei Kleinkindern wenig überraschend. Offen bleibt jedoch, inwiefern ältere Kinder das Freizeitverhalten der Eltern beeinflussen. Zum einen reduziert sich der Betreuungsaufwand mit dem Alter des Kindes erheblich (Walter/Künzler 2002), so dass wieder größere Freiräume für Freizeitaktivitäten entstehen, zum anderen ergeben sich durch Kinder informelle als auch institutionelle Gelegenheitsstrukturen für neue Sozialkontakte. Im Gegensatz zu früheren Studien wird in der vorliegenden Untersuchung ebenfalls die Situation von Vätern mit Kinder im Schul- und Jugendalter betrachtet.

Zweitens werden die unterschiedlichen Formen von Vaterschaft in früheren Studien kaum thematisiert. Hier dominiert zumeist implizit das Leitbild des biologisch-sozialen Vaters, womit vernachlässigt wird, dass sich im Zuge steigender Trennungs- und Scheidungsraten biologische und soziale Vaterschaft zusehends entkoppeln. Die Zunahme nicht- 
traditioneller Familienformen wird in der vorliegenden Studie explizit berücksichtigt, da innerhalb der Analysen ausdrücklich zwischen biologischen Vätern und Stiefvätern differenziert wird. Darüber hinaus geht dieser Beitrag der Frage nach, inwiefern zwischen ehelichen und nichtehelichen Familiengründungen Unterschiede in den Auswirkungen der Elternschaft bestehen.

Drittens berücksichtigt die vorliegende Studie das Geschlecht der Kinder. Verschiedene empirische Untersuchungen deuten darauf hin, dass die Geschlechterkomposition des Nachwuchses verschiedene familiale Ereignisse und Prozesse wie Eheschließung und Scheidung (Morgan et al. 1988; Morgan/Pollard 2002; Wagner 1997), die Beziehung der Eltern zueinander (Werneck 1997) sowie das parentale Engagement (Harris/Morgan 1991) beeinflusst. Wir erwarten daher, dass sich Väter von Jungen ebenfalls hinsichtlich ihrer Lebenszufriedenheit und ihren Freizeitaktivitäten von Vätern mit Töchtern unterscheiden.

Viertens werden die Auswirkungen der Vaterschaft auf das Wohlbefinden, die Freizeitaktivitäten und die Beziehung zur Herkunftsfamilie anhand eines prospektiven Designs untersucht. Frühere Studien sind häufig querschnittlich angelegt (Fthenakis/Minsel 2002) oder verzichten auf eine Vergleichsgruppe bestehend aus kinderlosen Männern (Fthenakis et al. 2002; Werneck 1998), wodurch Rückschlüsse auf die Kausalrichtung der beobachteten Zusammenhänge kaum möglich sind.

\section{Theoretische Überlegungen}

Eine theoretische Rahmung erfahren Studien zu familialen Übergängen und ihren Auswirkungen in dem Familien-Transitions-Ansatz (Cowan 1991; Fthenakis 1999). Diesem Ansatz zufolge geht der Übergang zur Elternschaft mit Veränderungen äußerer Art wie der Reorganisation des sozialen Netzwerkes, Rollenveränderungen sowie Restrukturierungen der persönlichen Kompetenzen als auch mit Veränderungen innerer Art im Selbstund Weltbild einher.

Transitionsphasen wie der Übergang zur Elternschaft bringen häufig eine Neugestaltung und Umstrukturierung der sozialen Bindungen mit sich. Zum einen werden durch die Familiengründung familiale Beziehungen wie die Beziehung zum Partner, zu den eigenen Eltern und anderen Verwandten gestärkt, zum anderen erfolgt eine Umgestaltung der auBerfamilialen Beziehungen, indem Freundschaften zu kinderlosen Personen vernachlässigt werden und verstärkt Kontakt zu anderen Eltern gesucht wird (Fthenakis 1999). Eng verbunden mit der Umstrukturierung sozialer Beziehungen erfolgt eine Reorganisation der Rollen. An die Rolle des Vaters und der Mutter sind normative Verhaltenserwartungen geknüpft, die das Fürsorgeverhalten, aber auch die Erwerbstätigkeit von Müttern und Vätern betreffen. Ferner erfolgt eine Restrukturierung der persönlichen Kompetenzen, die vor allem den Erwerb neuer Fertigkeiten und Kenntnisse zur Bewältigung familialer Aufgaben und Konflikte umfasst. Die inneren Veränderungen betreffen Modifikationen des Selbst- und Weltbildes: Indem bisherige Erwartungen bezüglich der Rollendefinitionen und des Beziehungsverhaltens hinterfragt und neu konzipiert werden, wird sowohl die eigene Identität umdefiniert als auch das subjektive Weltbild restrukturiert. 
Ausgehend von diesen Überlegungen werden im Folgenden mögliche Auswirkungen der Vaterschaft auf das Wohlbefinden, die Freizeitaktivitäten und die Beziehung zur Herkunftsfamilie diskutiert. Hieran anschließend skizzieren wir Unterschiede zwischen biologischen und sozialen Vätern sowie zwischen ehelichen und nicht-ehelichen Vaterschaften. Abschließend wird beleuchtet, inwiefern das Geschlecht des Kindes die Auswirkungen der Vaterschaft beeinflusst.

\section{Lebenszufriedenheit, Freizeitaktivitäten und familiale Beziehungen von Vätern}

Lebenszufriedenheit: Psychologische Studien zur Partnerschaftsentwicklung weisen darauf hin, dass nach der Familiengründung die Beziehungsqualität und die Partnerschaftszufriedenheit infolge starker Belastungen und der Einschränkung eigener Bedürfnisse deutlich nachlassen (Fthenakis et al. 2002; Schneider/Rost 1999; Werneck 1997). Allerdings entscheiden sich Paare oftmals für eine Familiengründung, weil sie Kinder als bereichernd für das eigene Leben empfinden. In modernen Gesellschaften vermitteln Kinder durch die emotionale Befriedigung der Eltern und die „Freunde am Kind“ in erster Linie einen psychischen Nutzen, wohingegen ökonomische Nutzenüberlegungen wie die Mithilfe im Haushalt oder Unterstützungsleistungen im Alter als auch der sozial-normative Nutzen des Statusgewinns durch die Elternschaft die Fertilitätsentscheidung kaum oder nur geringfügig beeinflussen sollten (Nauck 2001; Huinink 2000). Demnach ist infolge der Familiengründung ein Anstieg des subjektiven Wohlbefindens zu erwarten. Fraglich ist jedoch, ob diese Zunahme der Lebenszufriedenheit nachhaltig ist oder eher temporären Charakter hat. So postulieren die in der Zufriedenheitsforschung dominanten Erklärungsansätze - die Adaptation-Level-Theorie (Brickman/Campell 1971) und die Set-PointTheorie (Heady/Wearing 1989) -, dass bedeutsame Lebensereignisse das subjektive Wohlbefinden nicht dauerhaft verändern können und nach einiger Zeit eine Annäherung an das ursprüngliche Zufriedenheitsniveau erfolgt. Einschlägige Studien zur Lebenszufriedenheit zeigen in der Tat, dass familiale Ereignisse wie die Heirat lediglich in den ersten Ehejahren das Zufriedenheitsniveau positiv beeinflussen (Stutzer/Frey 2006) und auch negative Erlebnisse wie Scheidung oder Tod des Partners einen relativ kurzfristigen Effekt auf das subjektive Wohlbefinden haben (Andreß/Bröckel 2007; Lucas et al. 2003). Eine erhöhte Lebenszufriedenheit sollte sich daher vor allem in den ersten Jahren nach der Familienbildung zeigen.

Freizeitgestaltung: Im Zuge der Familiengründung ist eine Umstrukturierung der Freizeit in verschiedener Hinsicht zu erwarten. Einerseits schränkt der Übergang zur Vaterschaft die Möglichkeiten der Freizeitgestaltung ein, da mehr Zeit für die Hausarbeit und Kinderbetreuung verwendet wird. Gerade junge Eltern verbringen einen Großteil ihrer Freizeit zu Hause, wodurch außerhäusliche Sozialkontakte und die Teilnahme am kulturellen Leben nachlassen (Rost/Schneider 1994).

Anderseits aber ergeben sich im Zuge der Familiengründung Gelegenheitsstrukturen für neue Sozialkontakte und soziales Engagement. So erfordert die institutionelle Einbindung des Kindes in den Kindergarten, die Schule oder den Sportverein oftmals ein bürgerschaftliches Engagement der Väter und Mütter und schafft neue Kontaktmöglichkeiten zu anderen Eltern. In der Tat weisen US-amerikanische Studien darauf hin, dass Väter 
häufiger in sozialen und politischen Verbänden aktiv sind als kinderlose Männer und sich oftmals an der Elternarbeit beteiligen (Eggebeen/Knoester 2001; Knoester/Eggebeen 2006). Ebenfalls berichten Väter eine höhere Teilnahme an religiösen Aktivitäten als Männer ohne Kinder (Umberson 1987). Wir gehen daher davon aus, dass auch in Deutschland der Übergang zur Vaterschaft eine stärkere Teilnahme an bürgerschaftlichen und religiösen Aktivitäten bewirkt.

Beziehung zur Herkunftsfamilie: Die familiale Situation hat einen nicht unerheblichen Einfluss auf die Beziehung zu den eigenen Eltern (Szydlik/Schupp 1998). Der Übergang zur Elternschaft kann sich aus zwei Gründen positiv auf die Generationenbeziehung auswirken. Erstens nehmen junge Eltern oftmals Unterstützungsleistungen etwa in Form der Kinderbetreuung in Anspruch, wodurch sich die Kontaktdichte erhöht, die Qualität der Beziehung zu den eigenen Eltern jedoch nur bedingt verbessert (Rost/Schneider 1994). Zweitens bauen Großeltern in der Regel eine emotionale Bindung zu ihren Enkeln auf und vertiefen dadurch das Verhältnis zu ihren Kindern (Szydlik/Schupp 1998). Zwar weist die einschlägige Literatur darauf hin, dass zumeist Frauen die verwandtschaftlichen Beziehungen pflegen (Wagner 2002) und dass das Eltern-Sohn-Verhältnis von familialen Ereignissen tendenziell weniger beeinflusst wird als das Eltern-Tochter Verhältnis - dennoch dürften Familiengründungen auch das Verhältnis der Männer zu ihren Eltern verändern. Zum einen sollte sich die Kontakthäufigkeit - etwa in Form von gegenseitigen Besuchen - erhöhen, zum anderen ist zu erwarten, dass die Beziehung der Väter zu den eigenen Eltern enger wird.

\section{Formen der Vaterschaft}

Biologische Väter und soziale Väter unterscheiden sich deutlich in ihrem Verständnis von Vaterschaft (Döring 2002; Wilk 2002). Der Stiefvater fungiert zumeist nicht als Ersatzvater, sondern wird häufig als eine Art Zusatzvater gesehen, der die Vaterrolle nicht vollständig übernimmt. Obwohl Stiefväter oftmals eine egalitäre Verteilung von Aufgaben und Funktionen befürworten, verbleibt die Zuständigkeit für die Kinder häufig bei der Mutter. Offenbar können oder wollen Stiefväter nicht in gleichem Maße wie biologischsoziale Väter Erziehungsaufgaben und Verantwortung bezüglich der Kinder übernehmen. Ebenfalls dürften die gesellschaftlichen Erwartungen an das parentale Engagement bei Stiefvätern deutlich geringer ausfallen als bei biologischen Vätern (Fine 1995).

Gemäß der Studie von Döring (2002) versteht sich der Großteil der Stiefväter als „Freund-Stiefvater“, der zwar bisweilen die Rolle des Vaters nach außen ausübt und auch väterliche Aufgaben wahrnimmt, sich jedoch nicht als Vater, sondern als zusätzliche Bezugsperson für die Kindern sieht. Wilk (2002) weist ferner darauf hin, dass die StiefvaterKind-Beziehung im Vergleich zur Vater-Kind-Beziehung durch weniger emotionale Nähe, Gemeinsamkeit und Unterstützung gekennzeichnet ist. Demzufolge sollte sich die soziale Vaterschaft schwächer auf das Wohlbefinden und das Alltagshandeln auswirken als die biologische Vaterschaft. Ähnlich ist anzunehmen, dass Großeltern zu biologischen Kindern ihrer Söhne eine innigere Beziehung aufbauen als zu Stiefenkeln und folglich eine engere Generationenbeziehung zwischen Eltern und Großeltern dann besteht, wenn biologische Kinder vorhanden sind. 
Ferner können die Auswirkungen des Übergangs zur Elternschaft mit dem Institutionalisierungsgrad der Partnerschaft variieren. Verheiratete und kohabitierende Paare unterscheiden sich hinsichtlich verschiedener Aspekte, die belastend oder erleichternd auf die Bewältigung der Familiengründung einwirken. Zum einen bestehen zwischen Personen in Ehen und Lebensgemeinschaften deutliche Differenzen hinsichtlich des familialen Alltagshandelns und familialer Vorstellungen. Empirische Studien weisen insbesondere auf die Prävalenz eines weniger traditionellen Rollenverständnisses bei Personen in nichtehelichen Partnerschaften (Clarkberg et al. 1995; Seltzer 2000) und einer egalitäreren Aufgabenverteilung bei unverheirateten Paaren hin (Klaus/Steinbach 2002; Wengler et al. 2008). Dies führt zu der Erwartung, dass sich Väter in nichtehelichen Lebensgemeinschaften stärker in der Familienarbeit engagieren (Walter/Künzler 2002) sowie eine engere Beziehung zum Kind aufbauen und damit eine höhere Lebenszufriedenheit erfahren als verheiratete Väter. Anderseits sind ungeplante Familiengründungen, die sich stark negativ auf die elterliche Beziehungsqualität und die subjektiv wahrgenommene Belastung auswirken (Reichle 2002; Werneck 1997), in nichtehelichen Lebensgemeinschaften deutlich überrepräsentiert (Rupp 1998). Zwar sind ungeplante Kinder nicht generell unerwünscht, allerdings steht die Mehrheit der betroffenen Männer der bevorstehenden ungeplanten Vaterschaft zurückhaltend oder ablehnend gegenüber (Rupp 1998). Da die Erwünschtheit der Elternschaft stark mit der Partnerschaftsform korreliert, könnten unverheiratete Männer im Vergleich zu verheirateten Vätern die Familiengründung als belastender empfinden und daher ein niedrigeres Zufriedenheitsniveau sowie ein geringeres elterliches Engagement aufweisen.

\section{Geschlecht des Kindes}

Die Überlegungen des „Value of Children“-Ansatzes legen nahe, dass Söhne und Töchter auch in modernen Gesellschaften einen geschlechtsspezifischen psychischen und sozialnormativen Nutzen für ihre Eltern stiften (s. Nauck 2001). Da Söhnen oftmals die Funktion des Stammhalters zukommt, fällt der sozial-normative Nutzen für beide Elternteile bei männlichen Nachkommen höher aus als bei weiblichen. Zum anderen ist der psychische Nutzen der Eltern bei gleichgeschlechtlichen Kindern größer als bei gegengeschlechtlichen Dyaden, da häufiger ähnliche Interessen und Hobbys bestehen. Diese Überlegungen lassen erwarten, dass Väter eine stärkere Präferenz für Jungen entwickeln.

Vorhandene empirische Analysen zum Kinderwunsch und Fertilitätsverhalten haben die Existenz einer Präferenz für Söhne bislang kaum bestätigen können (siehe etwa Brockmann 2001; Hank/Kohler 2003). Allerdings zeigen verschiedene Studien, dass familiale Entscheidungs- und Entwicklungsprozesse durch die Geschlechterkomposition des Nachwuchses geprägt werden. Eine besonders hohe Aufmerksamkeit wurde der Scheidungswahrscheinlichkeit in Abhängigkeit vom Geschlecht der Kinder gewidmet; hier deuten mehrere Untersuchungen darauf hin, dass Väter und Mütter von Söhnen einem niedrigeren Scheidungsrisiko unterliegen als Eltern mit Töchtern (für die USA: Morgan et al. 1988; Morgan/Pollard 2002; für Deutschland: Wagner 1997)². Ebenfalls beob-

2 Siehe jedoch die Kritik von Diekmann und Schmidheiny (2004). 
achten Dahl und Moretti (2008) sowie Lundberg und Rose (2003) für die USA eine höhere Heiratswahrscheinlichkeit von kohabitierenden Paaren, wenn ein Junge anstatt eines Mädchens geboren wird. US-amerikanische Analysen zur Zeitverwendung zeigen ferner, dass Väter mehr Zeit mit ihren Söhnen als mit ihren Töchtern verbringen. Dies gilt sowohl für Freizeitaktivitäten als auch für Betreuungs- und Erziehungsaufgaben (siehe etwa Lundberg et al. 2007; Morgan et al. 1988; Yeung et al. 2001). Ausgehend von diesen Befunden erwarten wir, dass Söhne einen stärkeren Effekt auf die Lebenszufriedenheit und die Lebensführung ihrer Väter ausüben, als Töchter.

\section{Datenbasis und Methode}

\section{Daten}

Als Datengrundlage für die Analysen dient das Sozio-oekonomische Panel (SOEP). Das SOEP (Wagner et al. 2007) ist eine seit 1984 jährlich durchgeführte Wiederholungsbefragung, an der aktuell etwa 21.200 Personen aus 11.700 Haushalten teilnehmen. Da etliche für die hier behandelte Fragestellung relevante Informationen in den 1980er Jahren nicht erhoben wurden, beschränkt sich die vorliegende Untersuchung auf die Daten der Jahre 1990 bis 2007. Ferner werden nur Respondenten berücksichtigt, die in den alten Bundesländern leben. Diese Eingrenzung wurde aufgrund zweier Sachverhalte vorgenommen. Zum einen wird der Ehe und der nichtehelichen Lebensgemeinschaft in beiden Landesteilen ein unterschiedlicher Stellenwert beigemessen. Im Gegensatz zu den alten Bundesländern stellen nichteheliche Partnerschaften in den neuen Ländern eine dauerhafte Alternative zur Ehe dar, sodass hier Familiengründungen außerhalb der Ehe einen anderen Stellenwert haben als in den alten Bundesländern (Rupp 1998: 42). Zum anderen liegen Informationen über die Beziehung zu den eigenen Eltern und zu Freizeitaktivitäten für die neuen Bundesländer erst ab 1995 und teilweise nur für zwei Erhebungswellen vor, wodurch die Längsschnittbetrachtung deutlich erschwert wird. Ferner beschränkt sich die Untersuchung auf Respondenten zwischen 18 und 60 Jahren, die gemeinsam mit einer Ehe- oder Lebenspartnerin in einem Haushalt leben. Da uns primär die Situation der Väter von minderjährigen Kindern interessiert, werden Väter mit volljährigen Kindern von der Analyse ausgeschlossen. Die Auswirkungen des Geschlechts des Kindes auf die Lebenszufriedenheit, die Freizeitaktivitäten und die Beziehung zu den eigenen Eltern werden anhand eines Subsamples untersucht. Um eventuell differentielle Effekte von Jungen und Mädchen möglichst präzise abbilden zu können, beschränkt sich dieser Analyseschritt auf kinderlose Paare sowie Paare mit genau einem Kind.

\section{Variablen}

Als abhängige Variablen werden die Lebenszufriedenheit, verschiedene Freizeitaktivitäten sowie die Beziehung zu den eigenen Eltern berücksichtigt. Die Lebenszufriedenheit wird anhand einer elfstufigen Skala ( $0=$ ganz und gar unzufrieden, $10=$ ganz und gar zufrieden) erfasst. Der Fragenkatalog des SOEP zum Freizeitverhalten umfasst verschiedene 
Aktivitäten, von denen in den folgenden Analysen „Essen und Trinken gehen (Café, Kneipe, Restaurant)“, „gegenseitige Besuche von Nachbarn, Freunden und Bekannten“, „gegenseitige Besuche von Familienangehörigen oder Verwandten“, „Ehrenamtliche Tätigkeiten in Vereinen, Verbänden oder sozialen Diensten“, „Kirchgang, Besuch von religiösen Veranstaltungen“ sowie „Besuch von Sportveranstaltungen“ betrachtet werden. Die Antwortvorgaben umfassen „täglich“, „mindestens einmal pro Woche“, mindestens einmal im Monat“, „seltener“ und ,nie“. Die Freizeitaktivitäten wurden in dieser Form bislang in vier Wellen erhoben (1990, 1995, 1998, 2003).

Die Enge der Beziehung zur Mutter und zum Vater wird im SOEP anhand der Antwortvorgaben ,sehr eng“, „,eng“, „mittel“, „nur flüchtig“ und „überhaupt keine Beziehung“ erfasst. Die Qualität der intergenerationalen Beziehung wird nur erfragt, wenn die Mutter bzw. der Vater nicht mit der Befragungsperson im gleichen Haushalt lebt. Informationen über die Enge der Beziehung zu den Eltern liegen für drei Befragungswellen vor (1991, 1996, 2001).

Als zentrale unabhängige Variable fungiert die familiale Situation. Die Erfassung der Familienstruktur aus Sicht der Männer wird dadurch erschwert, dass eine retrospektiv erhobene Geburtsbiographie für den größten Teil des Untersuchungszeitraums lediglich für Frauen, nicht aber für Männer vorliegt. Daher erfassen wir die Elternschaft der Männer anhand der Geburtsangaben ihrer Lebens- und Ehepartnerinnen. Dies hat zur Folge, dass Kinder, die nicht im väterlichen Haushalt leben, unberücksichtigt bleiben. Diese Einschränkung stellt für die Analysen jedoch kein gravierendes Problem dar, da außerhalb des Haushalts lebende Kinder deutlich schwächer das Alltagshandeln der Männer beeinflussen als anwesende Kinder (Eggebeen/Knoester 2001).

Da die Lebenszufriedenheit und das Freizeitverhalten vermutlich stärker durch die Altersstruktur der Kinder als durch die Anzahl an Kindern im Haushalt geprägt werden, bilden wir die familiale Situation anhand des Alters des ältesten Kindes ab. Wir unterscheiden zwischen kinderlosen Männern, Vätern, deren ältestes Kind nicht älter als 6 Jahre alt ist, Väter, deren ältestes Kind zwischen 7 und 13 Jahre alt ist sowie Vätern, deren ältestes Kind zwischen 14 und 17 Jahre alt ist. ${ }^{3}$ In den Analysen zur Auswirkung des Geschlechts des Kindes auf das väterliche Verhalten wird die familiale Situation anhand von zwei binär kodierten Variablen (1 Junge vs. kein Junge; 1 Mädchen vs. kein Mädchen) abgebildet.

Ferner wird die Form der Vaterschaft berücksichtigt, indem wir zwischen biologischen Kindern und Stiefkindern unterscheiden. Weil im SOEP keine präzisen Angaben zum Verwandtschaftsverhältnis zwischen Vätern und ihren (Stief-)Kindern vorliegen, wird die Art der verwandtschaftlichen Beziehung anhand von Informationen über die Entwicklung der Haushaltszusammensetzung und der Ehebiografie beider Partner rekonstruiert. Auf diese Weise lässt sich das Verwandtschaftsverhältnis bei $98 \%$ der Kinder mit hoher Sicherheit bestimmen. Biologische Kinder werden weiterhin dahingehend unter-

3 Einige Aktivitäten, insbesondere abendliche Freizeitaktivitäten, werden sicherlich eher durch das Alter des jüngsten Kindes beeinflusst. Aus diesem Grund wurde in weiteren Modellspezifikationen die familiale Situation über das Alter des jüngsten Kindes abgebildet. Die Ergebnisse beider Modellspezifikationen unterscheiden sich nur marginal voneinander, sodass der Übersichtlichkeit halber in allen Modellen die Familienstruktur anhand des Alters des ältesten Kindes berücksichtigt wird. 
schieden, ob die Eltern zum Zeitpunkt der Familiengründung verheiratet waren oder nicht. Wir unterscheiden somit zwischen folgenden Gruppen: Väter von biologischen Kindern und Familiengründung im Rahmen einer Ehe (Referenzgruppe), Väter von biologischen Kindern und Familiengründung im Rahmen einer nichtehelichen Partnerschaft, Väter von ausschließlich Stiefkindern. ${ }^{4}$ Weil alle Modelle bereits das Vorhandensein von Kindern über die Alterstruktur umfassen, werden das Verwandtschaftsverhältnis und der Familienstand zum Zeitpunkt der Familiengründung anhand von Interaktionstermen berücksichtigt.

Ferner werden verschiedene Kontrollvariablen in die Modelle aufgenommen. Da nichteheliche Familiengründungen und die Stiefvaterschaft in jüngeren Kohorten, die eine modernere Einstellung zur Vaterschaft aufweisen, häufiger auftreten als in den älteren Geburtskohorten, besteht die Gefahr, dass die Effekte der nichtehelichen Familiengründung sowie der Stiefvaterschaft einerseits und die Effekte der kohortenbedingten Unterschiede in der Vaterrolle anderseits konfundieren. Zwecks Berücksichtigung möglicher Kohorteneffekte wird daher die Geburtskohorte (bis einschließlich 1960 geboren vs. später als 1960 geboren) als weitere Kontrollvariable in das Modell aufgenommen.

Zur Vermeidung von Scheinkorrelationen infolge unbeobachteter Heterogenität werden ferner der Erwerbsstatus der Männer als auch der ihrer Partnerinnen (vollzeitbeschäftigt, teilzeitbeschäftigt, nichterwerbstätig) und das Qualifikationsniveau (keine Ausbildung, Berufsausbildung, Fachhochschul- und Hochschulabschluss) in die Analyse einbezogen, da die Erwerbssituation und das Bildungsniveau sowohl mit dem Fertilitätsverhalten als auch mit den hier betrachteten abhängigen Variablen korrelieren dürften. Diese Variablen fungieren lediglich als Kontrollvariablen, daher werden die Koeffizienten nicht inhaltlich interpretiert. Um eine möglichst präzise Kontrolle der Alterseffekte zu erzielen, wird in den Analysen schließlich das Alter der Respondenten anhand eines Sets von Dummy-Variablen für jedes Lebensjahr berücksichtigt.

\section{Analyseverfahren}

Zur Analyse von Längsschnittdaten eignen sich insbesondere Panelregressionsverfahren (Brüderl 2010). Die Angaben zur Lebenszufriedenheit können als metrisch skaliert eingestuft werden, daher nutzen wir für diesen Analyseschritt das Fixed-Effects (FE) Regressionsmodell. Die Antwortvorhaben hinsichtlich des Freizeitverhaltens sowie der Beziehung zu den Eltern sind ordinal skaliert und werden anhand des Ordered Random-Effects (RE) Regressionsmodells ${ }^{5}$ analysiert.

Die FE-Regression unterscheidet sich von der linearen Regression darin, dass die abhängige Variable als auch die unabhängigen Variablen um den individuellen Mittelwert bereinigt werden. Durch die Mittelwertbereinigung greifen die FE-Schätzungen zur Bestimmung der Koeffizienten lediglich auf die intra-individuelle Varianz zurück. Anhand

4 Männer, die sowohl mit biologischen als auch mit Stiefkindern zusammenleben, werden ab dem Jahr der Geburt des ersten biologischen Kindes als „,biologische Väter“ kategorisiert.

5 Die FE-Regression hat bei kategorialen Responsevariablen den Nachteil, dass alle Fälle von der Analyse ausgeschlossen werden müssen, bei denen die abhängige Variable über die Zeit konstant ist. Aus diesem Grund wird hier ein RE-Regressionsmodell angewandt. 
dieser Vorgehensweise wird unbeobachtete Heterogenität, die Selektionseffekten zugrunde liegt und Endogenität zwischen den unabhängigen Variablen und dem Störterm verursacht, eliminiert. Die Koeffizienten der FE-Regression werden auf die gleiche Art interpretiert wie die einer linearen Regression. Bei der RE-Regression erfolgt ebenfalls wie bei der FE-Regression eine Subtraktion der individuellen Mittelwerte, jedoch wird im Gegensatz zur FE-Regression der unbeobachtete Effekt nicht eliminiert, sondern als Zufallsvariable geschätzt. Weiterhin unterscheidet sich die RE-Regression von der FE-Regression darin, dass allein bei der RE-Regression zeitkonstante unabhängige Variablen in das Modell aufgenommen werden können. Daher wird die Kohortenzugehörigkeit lediglich bei der Analyse der Freizeitaktivitäten und der Beziehung zu den eigenen Eltern berücksichtigt (nicht aber bei der Analyse der Lebenszufriedenheit).

\section{Empirische Ergebnisse}

\section{Lebenszufriedenheit}

Wie die Ergebnisse in Tabelle 1 zeigen, wirkt sich der Übergang zur Vaterschaft positiv auf die Lebenszufriedenheit aus. Ein signifikanter Effekt ist allerdings allein bei Vätern zu beobachten, deren ältestes Kind maximal 6 Jahre alt ist, wohingegen Väter von Kindern über 6 Jahren kein höheres Zufriedenheitsniveau berichten als kinderlose Männer. Dieser Befund steht in Einklang mit den theoretischen Annahmen der Zufriedenheitsforschung, die lediglich temporäre Effekte von Lebensereignissen auf das Zufriedenheitsniveau prognostizieren. Entgegen unserer Annahme besteht kein signifikanter Unterschied zwischen Vätern von biologischen Kindern und Stiefvätern, jedoch verzeichnen Väter von nichtehelichen Kindern ein - schwach signifikant - höheres Zufriedenheitsniveau als Väter, bei denen die Familiengründung im Rahmen der Ehe erfolgte. Dieses Ergebnis entspricht der oben angeführten Annahme, dass kohabitierende Väter in einem höheren Maße familial engagiert sind und sich daher die Familienbildung bei ihnen stärker auf das Zufriedenheitsniveau auswirkt als bei verheirateten Männern. Die Überlegung, dass nichteheliche Familiengründungen aufgrund ihrer häufigen Ungeplantheit mit einer niedrigeren Lebenszufriedenheit im Vergleich zu ehelichen Familienbildungen einhergehen, wird dagegen nicht empirisch gestützt.

Jedoch verbirgt sich hinter diesem Befund möglicherweise ein Kohorteneffekt, da nichteheliche Familiengründungen verstärkt in jüngeren Geburtskohorten auftreten, die vermutlich aufgrund einer moderneren Einstellung zur Vaterschaft einen stärkeren Anstieg der Lebenszufriedenheit nach der Familiengründung verzeichnen als ältere Geburtskohorten. Der positive Koeffizient der nichtehelichen Familiengründung würde demnach auf nicht einen kausalen Effekt dieser Partnerschaftsform auf die Lebenszufriedenheit hinweisen, sondern die nichteheliche Familiengründung würde lediglich als Indikator für die Zugehörigkeit zu einer jüngeren Alterskohorte fungieren. Um diesen Erklärungsversuch empirisch zu überprüfen, wird die Kohortenzugehörigkeit „konstant gehalten“, indem Modell 1 getrennt für zwei Geburtskohorten (Geburtsjahr bis einschließlich 1960 und Geburtsjahr 1961 oder später) berechnet wird. Wenn der positive Koeffizient der nicht-ehelichen Familiengründung auf einem Kohorteneffekt beruht, dann müssten beide 
Modelle niedrige und nicht-signifikante Koeffizienten für diese Variable hervorbringen. Tatsächlich sind die die Koeffizienten für die nichteheliche Familiengründung in beiden Modellen relativ groß ohne jedoch ein akzeptables Signifikanzniveau zu erreichen ${ }^{6}$, sodass die Existenz eines Kohorteneffektes nicht völlig ausgeschlossen werden kann.

Tabelle 1: Determinanten der Lebenszufriedenheit (Fixed Effects Regression)

\begin{tabular}{|c|c|c|c|c|}
\hline & \multicolumn{2}{|l|}{ Modell 1} & \multicolumn{2}{|l|}{ Modell 2} \\
\hline & Koef. & Stdf. & Koef. & Stdf. \\
\hline Verheiratet $^{1}$ & $-0,036$ & 0,037 & $-0,020$ & 0,589 \\
\hline Ältestes Kind unter 7 Jahren ${ }^{2}$ & $0,093^{*}$ & 0,041 & & \\
\hline Ältestes Kind 7-13 Jahre ${ }^{2}$ & 0,073 & 0,050 & & \\
\hline Ältestes Kind 14-17 Jahre ${ }^{2}$ & 0,018 & 0,063 & & \\
\hline Stiefkinder ${ }^{3}$ & 0,102 & 0,096 & & \\
\hline Nichtehel. Familiengründung ${ }^{3}$ & $0,167^{+}$ & 0,098 & & \\
\hline Junge $^{2}$ & & & $0,176^{\star *}$ & 0,051 \\
\hline Mädchen ${ }^{2}$ & & & 0,076 & 0,053 \\
\hline Teilzeitbeschäftigt $^{4}$ & $-0,307^{\star *}$ & 0,059 & $-0,206^{\star \star}$ & 0,067 \\
\hline Nichterwerbstätig ${ }^{4}$ & $-0,901^{* *}$ & 0,034 & $-0,852^{* \star}$ & 0,040 \\
\hline Keine Ausbildung ${ }^{5}$ & $-0,025$ & 0,047 & $-0,082$ & 0,056 \\
\hline Fachhochschulabschluss ${ }^{5}$ & $-0,163^{*}$ & 0,0749 & $-0,153^{+}$ & 0,092 \\
\hline Hochschulabschluss $^{5}$ & $-0,030$ & 0,100 & 0,036 & 0,113 \\
\hline Partnerin teilzeitbeschäftigt ${ }^{6}$ & $-0,111^{* *}$ & 0,028 & $-0,111^{* *}$ & 0,032 \\
\hline Partnerin nichterwerbstätig ${ }^{6}$ & $-0,101^{* *}$ & 0,028 & $-0,090^{* *}$ & 0,031 \\
\hline Konstante & $7,592^{* *}$ & 0,051 & $8,890^{* *}$ & 0,178 \\
\hline \multicolumn{5}{|c|}{ Signifikanzniveau: ${ }^{* *}=p<0,01 ;{ }^{*}=p<0,05 ;^{+}=p<0,1$} \\
\hline Fallzahl (Personenjahre) & 34.545 & & 25.861 & \\
\hline $\mathrm{R}^{2}$ (within) & 0,05 & & 0,04 & \\
\hline
\end{tabular}

Ausprägungen der abhängigen Variablen: $0=$ ganz und gar unzufrieden; $10=$ ganz und gar zufrieden.

1) Referenzgruppe: nichteheliche Partnerschaft; 2) Ref.: kein Kind 3) Ref.: biologisches Kind mit ehelicher Familiengründung; 4) Ref.: vollzeitbeschäftigt; 5) Ref.: Berufsausbildung; 6) Ref.: Partnerin vollzeitbeschäftigt Koef. = Koeffizient; Stdf. = Standardfehler. Alle Modelle enthalten DummyVariablen für das jeweilige Lebensalter.

Quelle: SOEP 1990-2007, eigene Berechnungen.

Der in Modell 1 ausgewiesene Koeffizient für Väter mit einem Kind im Vorschulalter ist zwar statistisch signifikant, jedoch indiziert dessen geringe Höhe einen eher schwachen Effekt auf die Lebenszufriedenheit. Möglicherweise ist der niedrige Koeffizient darauf zurückzuführen, dass die familiale Situation nicht in adäquater Weise erfasst wird, da sich der positive Effekt der Vaterschaft auf die ersten Jahre unmittelbar nach der Geburt des Kindes beschränkt. Um präzisere Erkenntnisse über die Auswirkung der Familiengründung auf die Lebenszufriedenheit zu erlagen, wird in einem weiteren Schritt das Untersuchungssample auf den Zeitraum bis zum 6. Jahr nach der Familiengründung begrenzt. Den Zeitverlauf bilden wir anhand von Dummy-Variablen ab, wobei der gesamte Beobachtungszeitraum bis zum 3. Jahr vor der Familiengründung als Referenzkategorie dient. Die Koeffizienten der Jahres-Dummyvariablen sind grafisch in Abbildung 1 dargestellt.

6 Die Koeffizienten der nichtehelichen Familiengründung betragen: 0,355; T-Wert: 1,16 (geboren vor 1960) und 0,134; T-Wert: 1,29 (geboren 1960 oder später). 
Abbildung 1: Auswirkungen der Familiengründung (FG) auf die Lebenszufriedenheit

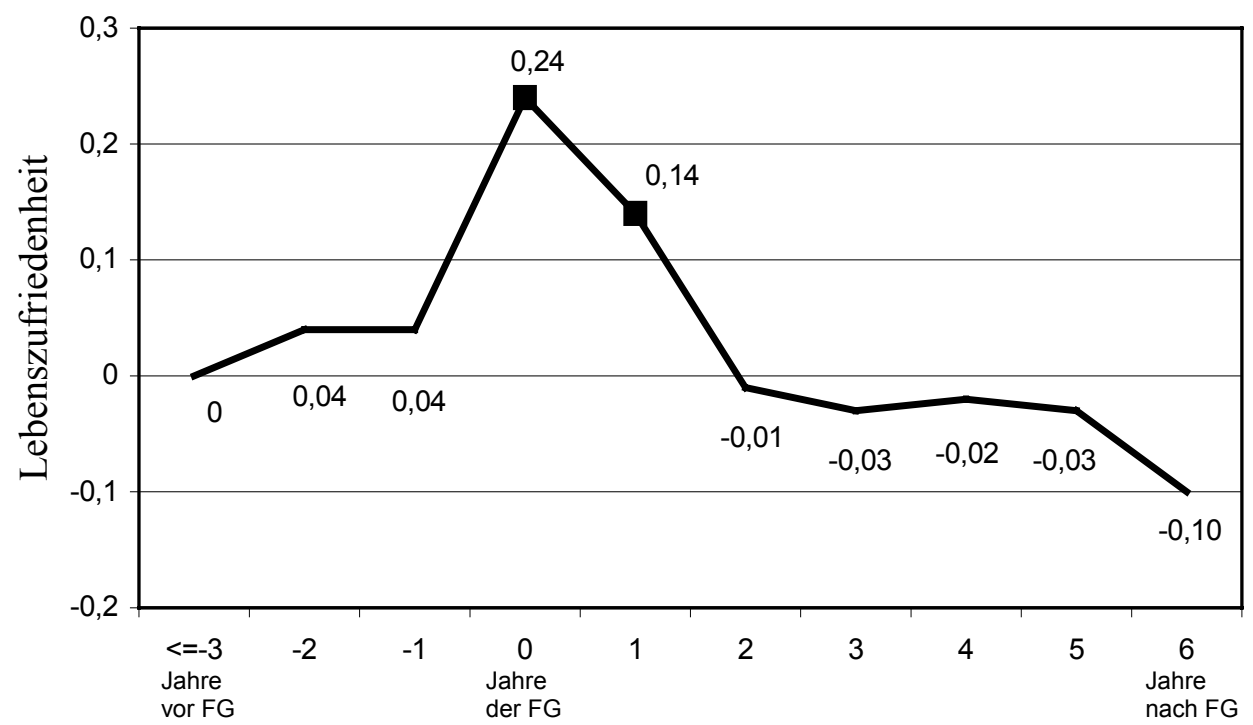

- = Koeffizient ist signifikant auf dem $5 \%$-Niveau

Kein weiterer Koeffizient ist auf dem $10 \%$-Niveau signifikant.

Referenzkategorie: Beobachtungszeitraum bis zum 3. Jahr vor der FG

Ein signifikant positiver Effekt der Familiengründung ist lediglich für das Jahr der Geburt des ersten Kindes und das Folgejahr zu erkennen. Die zu beobachtenden Auswirkungen sind zwar angesichts der elfstufigen Skala, die der Messung zugrunde liegt, noch immer nicht sonderlich stark ausgeprägt, jedoch fallen die Effekte deutlich höher aus als jene in Modell 1 und entsprechen ziemlich genau dem von Lucas et al. (2003) ermittelten Effekt der Eheschließung auf die Lebenszufriedenheit ${ }^{7}$.

In Modell 2 wird nun der Frage nachgegangen, inwiefern der Effekt der Vaterschaft auf die Lebenszufriedenheit mit dem Geschlecht des Kindes variiert. Entsprechend den oben getroffenen Annahmen zeigt sich ein besonders starker Effekt bei Vätern von Jungen, wohingegen die Geburt eines Mädchens die Lebenszufriedenheit nicht in signifikanter Weise beeinflusst. Der Unterschied zwischen beiden Koeffizienten ist zwar recht deutlich, allerdings ist die Differenz lediglich auf dem 11-Prozent-Niveau signifikant (TWert: 1,57). Insgesamt bekräftigt dieses Ergebnis jedoch die Annahme einer Geschlechterpräferenz für Jungen seitens der Väter.

\section{Freizeitverhalten}

Der Übergang zur Vaterschaft hat restringierende, aber auch positive Auswirkungen auf die Freizeitgestaltung (Tabelle 2). Wenig überraschend zeigen sich in Modell 1a negative Ef-

7 Die Studie von Lucas et al. (2003: 532) basiert ebenfalls auf den SOEP-Daten und berichtet einen Anstieg der Lebenszufriedenheit um 0,23 Punkte infolge der Eheschließung. 
fekte hinsichtlich zumeist abendlicher Freizeitaktivitäten wie dem Besuch von Restaurants, Kneipen oder Cafés. Anders als oben vermutet schwächt sich dieser Effekt nicht mit dem Alter der Kinder ab. Da in allen Modellen das Alter des Vaters kontrolliert wird, ist auszuschließen, dass der stark negative Effekt von Kindern im Jugendalter auf einem Alterseffekt der Väter beruht. Gegen die hier ausgewiesene Modellspezifikation lässt sich allerdings einwenden, dass die abendliche Freizeitgestaltung primär durch das Alter des jüngsten und nicht des ältesten Kindes beeinflusst wird. In einer weiteren Modellspezifikation wurde die familiale Situation daher anhand des Alters des jüngsten Kindes abgebildet; die dadurch erzielten Ergebnisse weichen nur marginal von den hier ausgewiesenen Befunden ab. Ferner zeigt sich in Modell 1a, dass Stiefväter ihre Restaurant-, Café- und Kneipen-Besuche in einem geringen Ausmaß einschränken als biologische Väter, jedoch ist der Interaktionsterm für die Stiefvaterschaft nur auf dem 10-Prozent-Niveau signifikant.

Negative Auswirkungen der Vaterschaft sind ebenfalls hinsichtlich der Häufigkeit gegenseitiger Besuche von Nachbarn, Freunden oder Bekannten (Modell 2a) zu beobachten, wobei der Effekt stärker ausfällt, wenn die Familiengründung im Rahmen einer nichtehelichen Lebensgemeinschaft erfolgte. Auch hier zeigt sich mit zunehmendem Alter der Kinder keine Abschwächung des negativen Effektes. Bei der Berücksichtigung der Geschlechtszugehörigkeit des Kindes in Modell 2b wird deutlich, dass die Vaterschaft nur dann die Besuchsfrequenz signifikant reduziert, wenn ein Mädchen geboren wurde, wohingegen Väter von Jungen ihre Nachbarn, Freude und Bekannte nicht signifikant seltener treffen als kinderlose Männer.

In Einklang mit den oben formulierten Erwartungen wirkt sich die Elternschaft positiv auf den Kontakt zu Familienangehörigen und Verwandten aus (Modell 3a). Eine signifikant erhöhte Besuchsfrequenz ist jedoch nur zu beobachten, solange sich das älteste Kind im Vorschulalter befindet. Auch hier wird die Effektstärke durch das Geschlecht des Kindes beeinflusst: Gemäß den Ergebnissen in Modell 3b berichten Väter von Jungen häufiger Verwandtschaftsbesuche als Väter von Mädchen. Insgesamt betrachtet deuten die Befunde der Modelle 2b und $3 \mathrm{~b}$ darauf hin, dass die Reduktion der Sozialkontakte bei Vätern von Mädchen stärker ausgeprägt ist als bei Vätern von Jungen.

Positive Effekte der Vaterschaft sind ferner hinsichtlich des bürgerschaftlichen und religiösen Engagements zu beobachten. So engagieren sich Väter von Kindern im Jugendalter stärker in Vereinen, Verbänden und sozialen Diensten als kinderlose Männer. Hier bestehen allerdings signifikante Unterschiede zwischen biologischen und sozialen Vätern: Eine erhöhte soziale Partizipation ist lediglich bei biologischen, nicht aber bei sozialen Vätern beobachtbar. Das schwache ehrenamtliche Engagement der Stiefväter ist vermutlich darauf zurückzuführen, dass die Vaterrolle nicht vollständig übernommen wird und viele Aufgaben - beispielsweise die Mitarbeit in Elterninitiativen oder schulischen Fördervereinen - vom biologischen Vater wahrgenommen werden. Ferner wirkt sich die Vaterschaft positiv auf den Kirchbesuch und die Teilnahme an religiösen Aktivitäten aus (Modell 5a). Auch hier beschränkt sich der Effekt der Elternschaft auf Väter von biologischen Kindern, wohingegen soziale Väter keine höhere religiöse Partizipation berichten als kinderlose Männer. Ebenfalls hat eine nichteheliche Familiengründung keinen positiven Effekt auf den Besuch religiöser Veranstaltungen. 


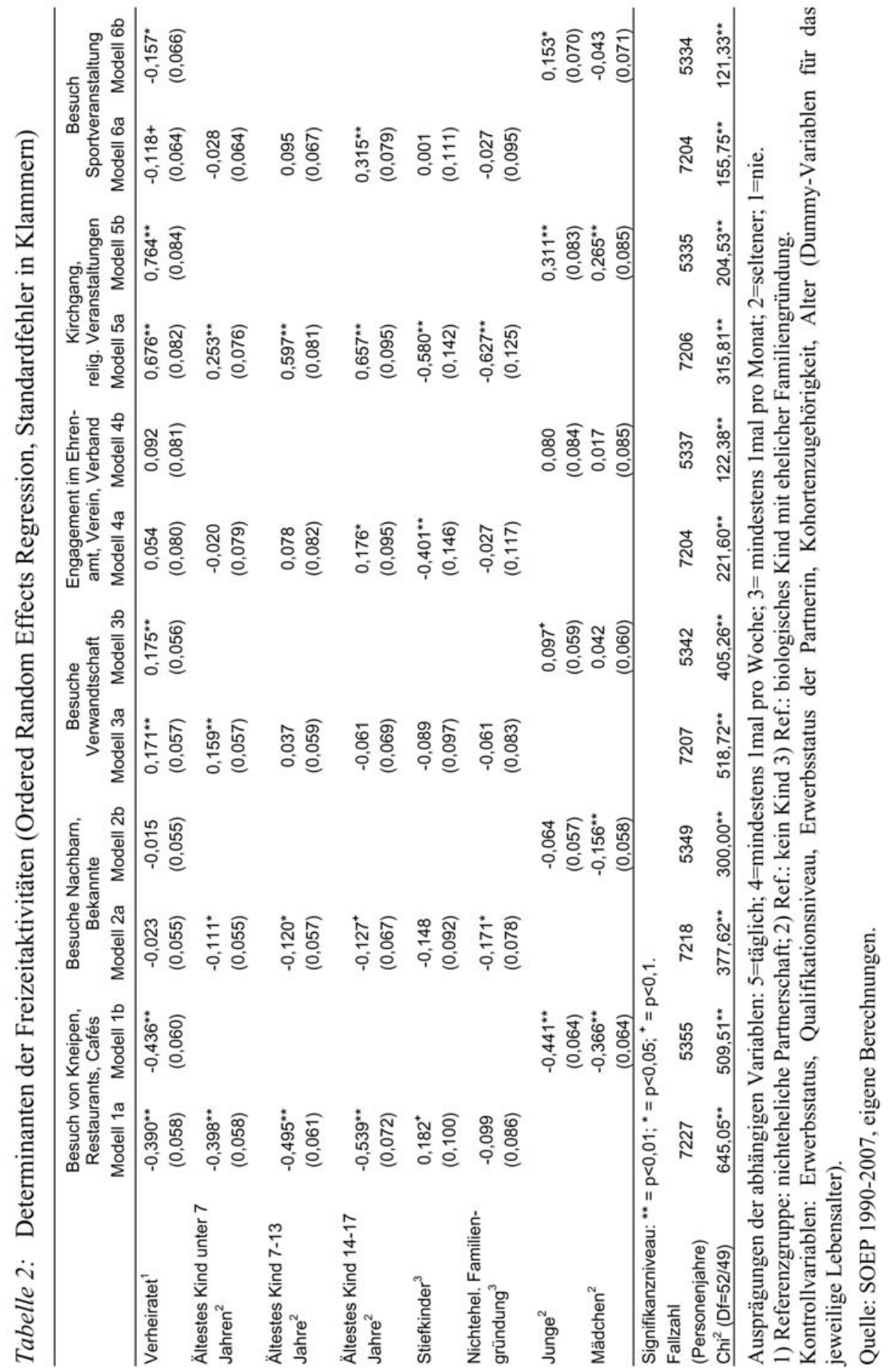


Schließlich wird der Besuch von Sportveranstaltungen durch die familiale Situation geprägt, indem Väter mit jugendlichen Kindern signifikant häufiger solche Veranstaltungen besuchen als kinderlose Männer (Modell 6a). Ein positiver Effekt ist allerdings nur bei Vätern von Söhnen zu beobachten, wohingegen Töchter die Besuchshäufigkeit von Sportveranstaltungen nicht signifikant beeinflussen (Modell 6b).

\section{Beziehung zur den eigenen Eltern}

Gemäß den oben durchgeführten Analysen erhöht sich im Zuge der Familiengründung die Häufigkeit verwandtschaftlicher Besuche (Tabelle 2, Modell 3a). Im Folgenden wird der Frage nachgegangen, ob sich der Übergang zur Vaterschaft nicht nur positiv auf die Kontaktdichte zu Familienangehörigen auswirkt, sondern auch die Beziehung zu den eigenen Eltern vertieft. Wie die Ergebnisse zeigen (Tabelle 3), wird das Verhältnis zum Vater lediglich in den ersten Jahren nach der Familiengründung als enger empfunden (Modell 1a), und auch dieser Effekt ist nur schwach signifikant. Die Beziehung zur Mutter bleibt dagegen gänzlich unverändert (Modell 2a).

Tabelle 3: Determinanten der Beziehungsenge zu den eigenen Eltern (Ordered Random Effects Regression, Standardfehler in Klammern)

\begin{tabular}{|c|c|c|c|c|}
\hline & \multicolumn{2}{|c|}{ Beziehung zum Vater } & \multicolumn{2}{|c|}{ Beziehung zur Mutter } \\
\hline & Modell 1a & Modell 1b & Modell 2a & Modell 2b \\
\hline Verheiratet $^{1}$ & $\begin{array}{c}0,058 \\
(0,086)\end{array}$ & $\begin{array}{c}0,068 \\
(0,085)\end{array}$ & $\begin{array}{c}0,030 \\
(0,075)\end{array}$ & $\begin{array}{c}0,057 \\
(0,073)\end{array}$ \\
\hline Ältestes Kind unter 7 Jahren² & $\begin{array}{l}0,152^{+} \\
(0,089)\end{array}$ & & $\begin{array}{c}0,092 \\
(0,077)\end{array}$ & \\
\hline Ältestes Kind 7-13 Jahre ${ }^{2}$ & $\begin{array}{c}0,129 \\
(0,097)\end{array}$ & & $\begin{array}{c}0,042 \\
(0,081)\end{array}$ & \\
\hline Ältestes Kind 14-17 Jahre ${ }^{2}$ & $\begin{array}{c}0,139 \\
(0,122)\end{array}$ & & $\begin{array}{c}0,120 \\
(0,098)\end{array}$ & \\
\hline Stiefkinder ${ }^{3}$ & $\begin{array}{l}-0,390^{*} \\
(0,156)\end{array}$ & & $\begin{array}{l}-0,290^{*} \\
(0,131)\end{array}$ & \\
\hline Nichtehel. Familiengründung ${ }^{3}$ & $\begin{array}{l}-0,198 \\
(0,123)\end{array}$ & & $\begin{array}{l}-0,136 \\
(0,107)\end{array}$ & \\
\hline Junge $^{2}$ & & $\begin{array}{c}0,055 \\
(0,094)\end{array}$ & & $\begin{array}{c}0,032 \\
(0,078)\end{array}$ \\
\hline Mädchen² & & $\begin{array}{c}0,123 \\
(0,096)\end{array}$ & & $\begin{array}{c}0,065 \\
(0,080) \\
\end{array}$ \\
\hline Signifikanzniveau: ${ }^{* *}=p<0,01$ & & & & \\
\hline
\end{tabular}

Ausprägungen der abhängigen Variablen: $5=$ sehr eng; 4=eng; 3=mittel; 2=flüchtig; 1=überhaupt keine Beziehung.

1) Referenzgruppe: nichteheliche Partnerschaft; 2) Ref.: kein Kind 3) Ref.: biologisches Kind mit ehelicher Familiengründung.

Kontrollvariablen: Erwerbsstatus, Qualifikationsniveau, Erwerbsstatus der Partnerin, Kohortenzugehörigkeit, Alter (Dummy-Variablen für das jeweilige Lebensalter).

Quelle: SOEP 1990-2007, eigene Berechnungen. 
Die ausgewiesenen Befunde deuten jedoch auf starke Unterschiede zwischen biologischen und sozialen Vätern hin. Männer, die zusammen mit Stiefkindern leben, berichten gar eine Verschlechterung der Beziehung zu den eigenen Eltern. Die Enge der intergenerationalen Beziehung ist jedoch nicht davon abhängig, ob sich die Familiengründung im Rahmen einer Ehe oder einer nichtehelichen Lebensgemeinschaft ereignet hat. Ebenfalls hat das Geschlecht des Kindes keinen Einfluss auf die Beziehungsenge.

\section{Diskussion und Zusammenfassung}

In der deutschsprachigen Familien- und Väterforschung herrscht generell die Auffassung vor, dass „,ie mit Kindern verbundenen ,Gewinne“ die ,Kosten“ überwiegen“ (Werneck 1997: 286). Allerdings wurden positive Auswirkungen der Familienbildung bislang selten explizit thematisiert; die meisten empirischen Studien fokussieren auf nachteilige Auswirkungen der Elternschaft und konstatieren zunehmende psychische Belastungen wie auch eine verringerte Partnerschaftszufriedenheit. Die hier präsentierten Ergebnisse deuten hingegen darauf hin, dass sich das subjektive Wohlbefinden durch die Vaterschaft erhöht. Für diese divergierenden Befunde - sinkende Partnerschaftszufriedenheit und steigende Lebenszufriedenheit - lassen sich verschiedene methodische Erklärungsmöglichkeiten anführen: Eine Ursache kann darin gesehen werden, dass deutsche Analysen zur Partnerschaftszufriedenheit oftmals deskriptiv durchgeführt werden (Rost/Schneider 1994) oder die Vergleichsgruppe der Kinderlosen unberücksichtigt bleibt (Fthenakis et al. 2002), wodurch mögliche Alterseffekte oder Effekte der Partnerschaftsdauer der familialen Situation zugeschrieben werden. Ferner beginnt die Messung der Partnerschaftszufriedenheit bei deutschsprachigen Studien zumeist erst unmittelbar vor der Geburt des ersten Kindes (z.B. Werneck 1998). Zu diesem Zeitpunkt sind die Lebenszufriedenheit und vermutlich auch die Zufriedenheit mit der Partnerschaft bereits angestiegen, was aufgrund des linkszensierten Beobachtungszeitraums in diesen Analysen nicht erfasst wird. Auch wenn den Ursachen der gegensätzlichen Auswirkungen der Familiengründung auf die Partnerschaftszufriedenheit und Beziehungsqualität einerseits und auf die Lebenszufriedenheit andererseits hier nicht nachgegangen werden kann, so machen die vorliegenden Ergebnisse zumindest deutlich, dass eventuellen Einbußen in der Beziehungsqualität und Partnerschaftszufriedenheit ein Anstieg der Lebenszufriedenheit gegenübersteht.

Hinsichtlich der Freizeitgestaltung zeigen die hier präsentierten Befunde, dass Vaterschaft nicht nur mit einem Rückzug ins Private einhergeht, sondern ebenfalls eine $\mathrm{Zu}$ nahme bestimmter außerhäuslicher Unternehmungen wie die Beteiligung an bürgerschaftlichen und religiösen Aktivitäten oder den Besuch von Sportveranstaltungen bewirkt. Solche positiven Auswirkungen auf die Freizeitgestaltung, die meist erst eintreten, wenn sich die Kinder im Schulalter befinden, wurden in früheren Studien weitgehend vernachlässigt. Der Übergang zur Vaterschaft impliziert folglich nicht nur eine „Verhäuslichung der Freizeit", sondern wirkt sich ebenfalls positiv auf die Freizeitgestaltung und das gesellschaftliche Engagement aus.

Ein weiteres zentrales Ergebnis der vorliegenden Untersuchung ist der zum Teil differentielle Effekt von biologischer und sozialer Vaterschaft. So erfahren biologische Väter eine signifikant engere Beziehung zu ihren eigenen Eltern als soziale Väter. Möglicher- 
weise führen traditionelle Vorstellungen der Großelterngeneration dazu, dass Familiengründungen außerhalb der klassischen Familienform nicht als solche akzeptiert werden und folglich keine Verbesserung der Generationenbeziehungen bewirken. Ebenfalls ist denkbar, dass Männer mit einer nur flüchtigen Beziehung zur Herkunftsfamilie eher zur Gründung einer Stieffamilie neigen als Männer mit starken familialen Bindungen. Ferner berichten soziale Väter ein geringeres ehrenamtliches Engagement und eine schwächere religiöse Partizipation als biologische Väter. Die geringere Teilnahme an bürgerschaftlichen und religiösen Aktivitäten deutet jedoch nicht zwangsläufig auf eine niedrigere Bereitschaft zur Übernahme elterlicher Verantwortung hin, sondern ist vermutlich auch darauf zurückzuführen, dass bestimmte Erziehungsaufgaben vom biologischen Vater wahrgenommen werden oder aber - aus rechtlichen Gründen - von der Mutter übernommen werden müssen. Insgesamt verdeutlichen die Befunde, dass soziale Väter ähnliche Auswirkungen der Elternschaft auf die Lebenszufriedenheit und das Alltagshandeln wie biologische Väter erfahren. Auch nichteheliche Familiengründungen unterscheiden sich in ihren Folgen kaum von Familiengründungen, die im Rahmen einer Ehe erfolgen.

Schließlich konnte gezeigt werden, dass die Auswirkungen der Vaterschaft partiell vom Geschlecht des Kindes abhängen. Väter von Söhnen verzeichnen eine höhere Lebenszufriedenheit und eine geringere Reduktion der Sozialkontakte als Väter mit Töchtern. Dabei stützen die geschlechterdivergenten Auswirkungen auf die Lebenszufriedenheit die These einer „Präferenz für Jungen“ seitens der Väter deutlich stärker als frühere Studien zum Effekt der Geschlechterkomposition der Kinder auf die Heirats- und Scheidungswahrscheinlichkeit. Die vorliegenden Ergebnisse legen die Frage nahe, inwiefern weitere Aspekte des familialen Verhaltens, wie etwa die väterliche Beteiligung an der Haus- und Familienarbeit durch das Geschlecht des Kindes geprägt werden und machen deutlich, dass die Geschlechterkomposition des Nachwuchses, aber auch der Kontext der Familiengründung in zukünftigen Untersuchungen ausführlicher als bislang berücksichtigt werden müssen.

\section{Literatur}

Andreß, H.-J. \& Bröckel, M. (2007). Income and life satisfaction after marital disruption in Germany. Journal of Marriage and the Family, 69 (2), S. 500-512.

Brickman, P. \&Campell, D.T. (1971). Hedonic relativism and planning the good society. In: M. H. Appley (Hrsg.), Adaptation level theory: a symposium. New York: Academic Press, S. 287-302.

Brockmann, H. (2001). Girls preferred? Changing patterns of sex preferences in the two German states. European Sociological Review, 17 (2), S. 189-202.

Brüderl, J. (2010). Kausalanalyse mit Paneldaten. In: H. Best \& C. Wolf (Hrsg.), Handbuch der sozialwissenschaftlichen Datenanalyse. Wiesbaden: VS Verlag

Cowan, P. A. (1991). Individual and family life transitions: a proposal for a new definition. In: P. A. Cowan \& M. Hetherington (Hrsg.), Family transitions. Hillsdale: Lawrence Erlbaum, S. 3-30.

Dahl, G. B. \& Moretti, E. (2008). The demand for sons. Review of Economic Studies, 75 (4), S. 1085-1120.

Diekmann, A. \& Schmidheiny, K. (2004). Do parents of girls have a higher risk of divorce? An eighteencountry study. Journal of Marriage and the Family, 66 (3), S. 651-660.

Döring, G. H. (2002). Soziale Vaterschaft in Stieffamilien. Imaginationen von reifendem Glück. Regensburg: Roderer.

Eggebeen, D. J. \& Knoester, C. (2001). Does fatherhood matter for men? Journal of Marriage and the Family, 63 (2), p. 381-393. 
Fine, M. A. (1995). The clarity and content of the stepparent role: A review of the literature. Journal of Divorce and Remarriage, 24 (1-2) , S. 19-34.

Fthenakis, W. E. (1999). Transitionspsychologische Grundlagen des Übergangs zur Elternschaft. In: Deutscher Familienverband (Hrsg.), Handbuch Elternbildung. Band 1. Wenn aus Partnern Eltern werden. Opladen: Leske + Budrich, S. 33-68.

Fthenakis, W. E., Kalicki, B. \& Peitz, G. (2002). Paare werden Eltern. Die Ergebnisse der LBS-Familien-Studie. Opladen: Leske + Budrich.

Fthenakis, W. E. \& Minsel, B. (2002). Die Rolle des Vaters in der Familie). Stuttgart: Kohlhammer ((Schriftenreihe des Bundesministeriums für Familie, Senioren, Frauen und Jugend, Bd. 213).

El-Giamal, M. (1997). Veränderung der Partnerschaftszufriedenheit und Streßbewältigung beim Übergang zur Elternschaft: Ein aktueller Literaturüberblick. Psychologie in Erziehung und Unterricht, 44 (4), S. 256-275.

Hank, K. \& Kohler, H.-P. (2003). Sex preferences for children revisited: New evidence from Germany. Population (English Edition), 58 (1), S. 133-143.

Harris, K. M. \& Morgan, S. P. (1991). Fathers, sons, and daughters: differential parental involvement in parenting. Journal of Marriage and the Family 53 (3), S. 531-544.

Heady, B. \& Wearing, A. (1989). Personality, life events, and subjective well-being: toward a dynamic equilibrium model. Journal of Personality and Social Psychology, 57 (4), S. 731-739.

Huinink, J. (2000). Soziologische Ansätze zur Bevölkerungsentwicklung. In: U. Mueller, B. Nauck \& A. Diekmann (Hrsg.), Handbuch der Demographie 1. Berlin: Springer, S, 339-386.

Kalicki, B., Peitz, G. \& Fthenakis, W. E. (2006). Die Bewältigung des Übergangs zur Vaterschaft. In: H. Werneck, M. Beham \& D. Palz (Hrsg.), Aktive Vaterschaft. Männer zwischen Familie und Beruf. Gießen: Psychosozial-Verlag, S. 80-93.

Klaus, D. \& Steinbach, A. (2002). Determinanten innerfamilialer Arbeitsteilung. Eine Betrachtung im Längsschnitt. Zeitschrift für Familienforschung, 14 (1), S. 21-43.

Knoester, C. \& Eggebeen, D. J. (2006). The effects of the transition to parenthood and subsequent children on men's well-being and social participation. Journal of Family Issues, 27 (11), S. 1532-1560.

Konietzka, D.\& Kreyenfeld, M. (2005). Nichteheliche Mutterschaft und soziale Ungleichheit: Zur sozioökonomischen Differenzierung der Familienformen in Ost- und Westdeutschland. Kölner Zeitschrift für Soziologie und Sozialpsychologie, 57 (1), S. 32-61.

Lucas, R. E., Clark, A. E., Georgellis, Y. \& Diener, E. (2003). Reexamining adaptation and the set point model of happiness: reactions to changes in marital status. Journal of Personality and Social Psychology, 84 (3), S. 527-539.

Lundberg, S., McLanahan, S. \& Rose, E. (2007). Child gender and father involvement in fragile families. Demography, 44 (1), S. 79-92.

Lundberg, S. \& Rose, E. (2003). Child gender and the transition to marriage. Demography 40 (2), S. 333-349.

Morgan, S. P., Lye, D. N. \& Condran, G. A. (1988). Sons, daughters, and the risk of marital disruption. American Journal of Sociology 94 (1), 110-129.

Morgan, S. P. \& Pollard, M.S. (2002). Do parents of girls really have a higher risk of divorce? Paper prepared for presentation at the 2002 annual meetings of the Population Association of America. May 8-10, Atlanta, Georgia.

Nauck, B. (2001). Der Wert von Kindern für ihre Eltern. „Value of Children“ als spezielle Handlungsstrategie des generativen Verhaltens und von Genernationenbeziehungen im interkulturellen Vergleich. Kölner Zeitschrift für Soziologie und Sozialpsychologie, 53 (3), S. 407-435.

Reichle, B. (2002). Partnerschaftsentwicklung junger Eltern: Wie sich aus der Bewältigung von Lebensveränderungen Probleme entwickeln. In: N. F. Schneider \& H. Matthias-Beck (Hrsg.), Elternschaft heute. Gesellschaftliche Rahmenbedingungen und individuelle Gestaltungsaufgaben. Opladen: Leske + Budrich, S. 75-93 (Sonderheft 2 der Zeitschrift für Familienforschung).

Reichle B. \& Werneck H. (1999). Übergang zur Elternschaft: Aktuelle Studien zur Bewältigung eines unterschätzen Lebensereignisses. Stuttgart: Enke. 
Rost, H. \& Schneider, N. F. (1994). Familiengründung und Auswirkungen der Elternschaft. Österreichische Zeitschrift für Soziologie, 19 (2), S. 34-57.

Rost, H. \& Schneider, N. F. (1995). Differentielle Elternschaft - Auswirkungen der ersten Geburt auf Männer und Frauen. In: B. Nauck \& C. Onnen-Isemann (Hrsg.), Familie im Brennpunkt von Wissenschaft und Forschung. Berlin: Luchterhand, S. 177-194.

Rupp, M. (1998). Lebensverhältnisse nichtverheirateter Frauen beim Übergang zur Elternschaft. In: W. Bien \& N. F. Schneider (Hrsg.), Kind ja - Ehe nein? Status und Wandel der Lebensverhältnisse von nichtehelichen Kindern und Kindern in nichtehelichen Lebensgemeinschaften. Opladen: Leske + Budrich, S. 35-50.

Schneider, N. F. \& Rost, H. (1999). Soziologische Aspekte des Übergangs zur Elternschaft. In: B. Reichle \& H. Werneck (Hrsg.), Übergang zur Elternschaft: Aktuelle Studien zur Bewältigung eines unterschätzten Lebensereignisses. Enke, S. 19-36.

Stutzer, A. \& Frey, B. S. (2006). Does marriage make people happy, or do happy people get married? Journal of Socio-Economics 35 (2), S. 326-347.

Szydlik, M. (1995). Die Enge der Beziehung zwischen erwachsenen Kindern und ihren Eltern - und umgekehrt. Zeitschrift für Soziologie 24 (2), S. 75-94.

Szydlik, M. \& Schupp, J. (1998). Stabilität und Wandel von Generationenbeziehungen. Zeitschrift für Soziologie 27 (4), S. 297-315.

Umberson, D. (1987). Family status and health behaviors: Social control as a dimension of social integration. Journal of Health and Social Behavior, 28 (3), S. 306-316.

Vaskovics, L. A., Rost, H. \& Rosenkranz, D. (2000). Was machen junge Väter mit ihrer Zeit? Die Zeitallokation junger Ehemänner im Übergang zur Elternschaft. Bamberg: Staatsinstitut für Familienforschung an der Universität Bamberg (ifb-Forschungsbericht 2).

Wagner, G. G., Frick, J. R. \& Schupp, J. (2007). The German Socio-Economic Panel Study (SOEP) Scope, evolution and enhancements. Schmollers Jahrbuch - Journal of Applied Social Science Studies 127 (1), S. 139-169.

Wagner, M. (1997). Scheidung in Ost- und Westdeutschland. Zum Verhältnis von Ehestabilität und Sozialstruktur seit den 30er Jahren. Frankfurt: Campus.

Wagner, M. (2002). Familie und soziales Netzwerk. In: R. Nave-Herz (Hrsg.), Kontinuität und Wandel der Familie in Deutschland. Stuttgart: Lucius \& Lucius, S. 227-251.

Walter, W. \& Künzler, J. (2002). Parentales Engagement. Mütter und Väter im Vergleich. In: N. F. Schneider \& H. Matthias-Beck (Hrsg.): Elternschaft heute. Gesellschaftliche Rahmenbedingungen und individuelle Gestaltungsaufgaben. Opladen: Leske + Budrich, S. 95-119 (Sonderheft 2 der Zeitschrift für Familienforschung).

Wengler, A., Trappe, H. \& Schmitt, C. (2008). Partnerschaftliche Arbeitsteilung und Elternschaft. Analysen zur Aufteilung von Hausarbeit und Elternaufgaben auf Basis des Generations and Gender Survey. Wiesbaden: Bundesinstitut für Bevölkerungsforschung (Materialen zur Bevölkerungswissenschaft Heft 127).

Werneck, H. (1997). Belastungsaspekte und Gratifikationen beim Übergang zur Vaterschaft. Psychologie in Erziehung und Unterricht, 44 (4), S. 276-288.

Werneck, H. (1998). Übergang zur Vaterschaft. Auf der Suche nach den „Neuen Vätern “. Wien: Springer.

Wilk, L. (2002). Die Gestaltung multipler Vaterschaft in Stieffamilien. In: S. Walper \& B. Schwarz (Hrsg.), Was wird aus den Kindern? Chancen und Risiken für die Entwicklung von Kindern aus Trennungs- und Stieffamilien. Weinheim: Juventa, S. 121-142.

Yeung, W. J., Sandberg, J. F., Davis-Kean, P. E. \& Hofferth, S. L. (2001). Children's time with fathers in intact families. Journal of Marriage and the Family, 63 (1), S. 136-154.

Eingereicht am/Submitted on: 07.02.2009

Angenommen am/Accepted on: 16.03.2010 
Anschrift des Autors/Address of the author:

Dr. Matthias Pollmann-Schult

Universität Bielefeld

Postfach 110131

33501 Bielefeld

E-mail: mpollmann-schult@uni-bielefeld.de 